\title{
Short-term escitalopram treatment and hippocampal volume
}

\author{
Beata R. Godlewska • Helge W. W. Hasselmann • Artemis Igoumenou • \\ Ray Norbury • Philip J. Cowen
}

Received: 19 June 2014 / Accepted: 4 October 2014 / Published online: 18 October 2014

(C) The Author(s) 2014. This article is published with open access at Springerlink.com

Major depression has been associated with volume loss in hippocampal regions in most but not all meta-analyses (Arnone et al. 2012: Bora et al. 2012). In animal studies, repeated antidepressant treatment increases hippocampal neurogenesis (Malberg et al. 2000) but there have been few longitudinal studies in depressed patients assessing the effects of antidepressant medication on hippocampal volume. Recently, however, Arnone and colleagues (2013) reported that 8-week treatment with the selective serotonin re-uptake inhibitor (SSRI) citalopram in 32 depressed patients produced a bilateral increase in hippocampal volume. The aim of the present study was to see if a similar effect could be demonstrated during short-term treatment with the SSRI, escitalopram.

We studied 33 unmedicated participants (mean age 29.9, range 20-61 years, 19 female) who were diagnosed using the Structured Interview for DSM-IV as having major depression. Participants were scanned before and during (mean duration 46 days, range 3866 days) treatment with escitalopram, $20 \mathrm{mg}$ daily. Twenty of the patients were antidepressant naïve. In the remainder, the mean drug-free interval was 117 weeks (range 8-468 weeks). Clinical assessment

B. R. Godlewska $(\bowtie) \cdot$ H. W. W. Hasselmann · P. J. Cowen Psychopharmacology Research Unit (PPRU),

University Department of Psychiatry, University of Oxford,

Oxford OX3 7JX, UK

e-mail: beata.godlewska@psych.ox.ac.uk

A. Igoumenou

Violence Prevention Research Unit (VPRU), Barts and The London

School of Medicine and Dentistry, Queen Mary University,

London EC1M 6BQ, UK

R. Norbury

Department of Psychology, University of Roehampton,

London SW15 5PU, UK included Beck Depression Inventory (BDI) and Hamilton Depression Rating Scale (HAM-D). Clinical response was classified as $\geq 50 \%$ symptom reduction from baseline on HAM-D. All participants gave informed consent to the study which was approved by the local ethics committee.

Participants were scanned at the University of Oxford Centre for Clinical Magnetic Resonance Research on a 3 Tesla Siemens Trio Scanner with a 12-channel head coil (Siemens, Germany) and $1-\mathrm{mm}^{3}$ voxel dimension (MPRAGE: repetition time $=2040 \mathrm{~ms}$, echo time $=4.68 \mathrm{~ms}$, flip angle $=8^{\circ}$, field of view $=256 \mathrm{~mm}$ ). All FMRIB Software Library (FSL) analyses followed default settings described at http://fsl.fmrib.ox.ac.uk/fsl/fslwiki/FSL. FSL-VBM smoothing was performed with an isotropic Gaussian kernel of $2 \mathrm{~mm}$. Hippocampi were automatically segmented using FSL-FIRST (Patenaude et al. 2011). A general linear model with 5000 permutations and threshold-free cluster enhancement was performed to assess voxel- and vertex-wise differences. FSL-FAST was used to estimate intracranial volume (ICV) by summing grey matter, white matter and cerebrospinal fluid volume and to normalise hippocampal volumes (hippocampal grey matter $\mathrm{mm}^{3} / \mathrm{ICV} \mathrm{cm}^{3}$ ). As VBM may be less sensitive to subcortical structures, we additionally conducted vertex analysis, using the FIRST tool in FSL, which is specially designed to analyse shape and volume differences in subcortical regions (Patenaude et al. 2011). Statistical analyses were performed using SPSS 21 software (IBM, USA) with $t$ tests and a two-tailed $\alpha=.05$.

Of the 33 participants, $20(61 \%)$ responded during escitalopram treatment (with 16 remitters following HAM-D $\leq 7$ criterion of remission). Overall, participants showed no change in hippocampal volume measures after escitalopram. Examining the treatment responders separately showed a trend towards reduced normalised hippocampal volume that was not explained by differences in ICV (Table 1). There was 
Table 1 Mean ( \pm standard deviation) depression scores, intracranial volume $\left(\mathrm{ICV}, \mathrm{cm}^{3}\right)$ and hippocampal volume $\left(\mathrm{mm}^{3}\right)$ before and during escitalopram treatment

\begin{tabular}{|c|c|c|c|c|}
\hline & Pre-treatment & On treatment ${ }^{\mathrm{a}}$ & $t$ & $p$ \\
\hline \multicolumn{5}{|c|}{ Entire sample $(n=33)$} \\
\hline BDI & $31.7 \pm 7.2$ & $15.2 \pm 11.4$ & 7.007 & $<.001$ \\
\hline HAM-D & $23.0 \pm 4.6$ & $9.5 \pm 7.8$ & 9.118 & $<.001$ \\
\hline $\mathrm{ICV}$ & $1478.6 \pm 158.6$ & $1482.5 \pm 129.7$ & -0.200 & .842 \\
\hline \multicolumn{5}{|c|}{ Left hippocampus } \\
\hline Absolute & $3887.06 \pm 553.09$ & $3897.09 \pm 545.42$ & -0.151 & .881 \\
\hline Normalised & $2.64 \pm 0.36$ & $2.64 \pm 0.37$ & 0.071 & .944 \\
\hline \multicolumn{5}{|c|}{ Right hippocampus } \\
\hline Absolute & $3874.94 \pm 485.70$ & $3869.55 \pm 462.71$ & 0.130 & .897 \\
\hline Normalised & $2.63 \pm 0.27$ & $2.62 \pm 0.30$ & 0.234 & .816 \\
\hline \multicolumn{5}{|c|}{ Responders $(n=20)$} \\
\hline BDI & $31.4 \pm 6.4$ & $9.3 \pm 7.5$ & 10.157 & $<.001$ \\
\hline HAM-D & $23.1 \pm 5.1$ & $4.4 \pm 3.9$ & 13.679 & $<.001$ \\
\hline $\mathrm{ICV}$ & $1450.6 \pm 125.0$ & $1483.2 \pm 128.1$ & -1.509 & .148 \\
\hline \multicolumn{5}{|c|}{ Left hippocampus } \\
\hline Absolute & $3910.13 \pm 661.48$ & $3819.95 \pm 604.22$ & 0.811 & .374 \\
\hline Normalised & $2.70 \pm 0.41$ & $2.59 \pm 0.41$ & 1.638 & .102 \\
\hline \multicolumn{5}{|c|}{ Right hippocampus } \\
\hline Absolute & $3837.22 \pm 526.87$ & $3784.96 \pm 538.83$ & 0.742 & .313 \\
\hline Normalised & $2.65 \pm 0.32$ & $2.56 \pm 0.37$ & 1.802 & .057 \\
\hline
\end{tabular}

${ }^{a}$ Mean treatment duration 46 days, range $38-66$ days
However, there is no reason to think that their effects on brain morphology would be different.

As noted above, there are relatively few longitudinal studies of the effects of antidepressant treatment on hippocampal volume and more are needed. However, while our findings are negative, they are in broad agreement with an earlier longitudinal investigation that reported no volumetric change in hippocampus after 7 months of successful SSRI treatment in 22 depressed patients (Vythilingam et al. 2004).

Acknowledgments The study was funded by Medical Research Council (MRC).

Conflict of interest Philip J Cowen has been a member of a paid advisory board for Lundbeck.

Open Access This article is distributed under the terms of the Creative Commons Attribution License which permits any use, distribution, and reproduction in any medium, provided the original author(s) and the source are credited.

\section{References}

Arnone D, McIntosh AM, Ebmeier KP, Munafò MR, Anderson IM (2012) Magnetic resonance imaging studies in unipolar depression: systematic review and meta-regression analyses. Eur Neuropsychopharmacol 22: 1-16. doi:10.1016/j.euroneuro.2011.05.003 
Arnone D, McKie S, Elliott R et al (2013) State-dependent changes in hippocampal grey matter in depression. Mol Psychiatry 18:12651272. doi: $10.1038 / \mathrm{mp} .2012 .150$

Bora E, Fornito A, Pantelis C, Yücel M (2012) Gray matter abnormalities in major depressive disorder: a meta-analysis of voxel based morphometry studies. J Affect Disord 138:9-18. doi:10.1016/j.jad.2011.03.049

Cipriani A, Furukawa TA, Salanti G, Geddes JR, Higgins JP, Churchill R, Watanabe N, Nakagawa A, Omori IM, McGuire H, Tansella M, Barbui C (2009) Comparative efficacy and acceptability of 12 newgeneration antidepressants: a multiple-treatments meta-analysis. Lancet 28:746-758. doi:10.1016/S0140-6736(09)60046-5
Malberg JE, Eisch AJ, Nestler EJ, Duman RS (2000) Chronic antidepressant treatment increases neurogenesis in adult rat hippocampus. $\mathrm{J}$ Neurosci 20:9104-9110

Patenaude B, Smith SM, Kennedy DN, Jenkinson M (2011) A Bayesian model of shape and appearance for subcortical brain segmentation. Neuroimage 56:907-922. doi:10.1016/j.neuroimage. 2011.02.046

Vythilingam M, Vermetten E, Anderson GM et al (2004) Hippocampal volume, memory, and cortisol status in major depressive disorder: effects of treatment. Biol Psychiatry 56:101-112. doi:10.1016/j. biopsych.2004.04.002 